\title{
Million Cells
}

National Cancer Institute

\section{Source}

National Cancer Institute. Million Cells. NCI Thesaurus. Code C67246.

A unit of cell count expressed in millions. 